\title{
Bimodal extinction without cross-modal extinction
}

\author{
Albrecht Werner Inhoff, Robert D Rafal, Michael J Posner
}

\begin{abstract}
Three patients with unilateral neurological injury were clinically examined. All showed consistent unilateral extinction in the tactile and visual modalities on simultaneous intramodal stimulation. There was virtually no evidence for crossmodal extinction, however, so that contralateral stimulation of one modality would have extinguished perception of ipsilateral stimuli in the other modality. It is concluded that the attentional system controlling the encoding of tactile and visual stimuli is not unified across the two sensory domains.
\end{abstract}

Extinction, the failure to perceive and report sensory signals contralateral to a cerebral lesion under conditions of double, simultaneous stimulation, is chiefly associated with parietal lobe lesions. Although "sensory competition" has been advanced as a pathophysiological mechanism for this sign, it has become clear that the disorder is at a higher level of information processing involving the neural control of attention. Recent studies have shown that visual extinction results from a deficit in disengaging attention from signals in the field ipsilateral to the lesion.

In a sequence of studies which examined the mechanism underlying extinction, Posner and various associates presented targets to ipsi- or contralateral visual fields of patients with unilateral parietal lesions. ${ }^{1-4}$ Under some conditions, ipsi- and contralateral cues were used which marked location of to-be-detected targets. Cues preceded targets by a variable time interval, ranging from $50 \mathrm{~ms}$ to $1000 \mathrm{~ms}$, and either correctly or incorrectly predicted the location of to-be-detected targets. The results showed a relatively unimpaired target detection performance when contralaterally presented targets were preceded by cues specifying the contralateral target location. When no cue was present, however, or when cues specified the ipsilateral target location, contralateral target detection revealed an "extinction-like" reaction time pattern-that is, when patients were instructed to attend to the contralateral visual field, target detection was intact but when they were instructed to attend to the ipsilateral visual field, target detection was impaired, showing the characteristic extinction-like pattern.

Clinical examination resulting from lesions of the parietal lobe may manifest itself either in a single sensory modality or in more than one modality. Ipsilateral visual stimulation may inhibit the concurrent or subsequent perception of visual targets in the contralateral visual field, and ipsilateral tactile (or auditory) stimulation may inhibit the perception of concurrent or subsequent contralateral tactile (or auditory) stimulation. Given that damage to parietal structures may affect the perception of stimuli across sensory modalities, it seems possible that visual and tactile perception are integrated in a cognitive system representing spatial relations in general. If this was the case, then spatial cues to one modality should affect the perception of spatial targets presented to another modality and cross-modal extinction should occur.

\section{Case reports}

We identified three patients who we could test for cross-modal extinction without confounding variables of primary sensory dysfunction, anosognosia, or other mental defects. The three patients reported here were fully alert, lucid and cooperative. All three had consistent bimodal extinction which was not accompanied by primary sensory defects, and each had only mild clinical neglect, requiring specific testing to identify.

\section{Patient 1}

This 36 year old right handed woman was hospitalised after the abrupt onset of a severe headache. CT revealed a small haematoma in the right superior temporal region with some extension of blood into the right posterior lateral ventricle. Cerebral arteriography on two occasions revealed no evidence of aneurysms. The second arteriogram, however, performed five days after the onset of symptoms, revealed severe spasm of the internal carotid artery and its branches. After this study the patient developed a left hemiparesis and inattention. The cranial nerves were normal except for a mild left facial weakness. There was a pronator drift of the left upper extremity with mild impairment of finger dexterity in that hand but no weakness. The deep tendon reflexes were normal and symmetrical with flexor plantar responses bilaterally. There was no visual field deficit on confrontation for detecting finger movement or counting fingers. Primary tactile sensation including light, touch, pain, temperature, pinprick, position, and vibratory sensations were all intact as was stereognosis. The patient's neurological deficit resolved quickly over the two days after her testing for within and cross modal extinction. 
Figure 1 Reconstruction of neurological injury of patient 2 , showing infarcted area in right parietal lobe.
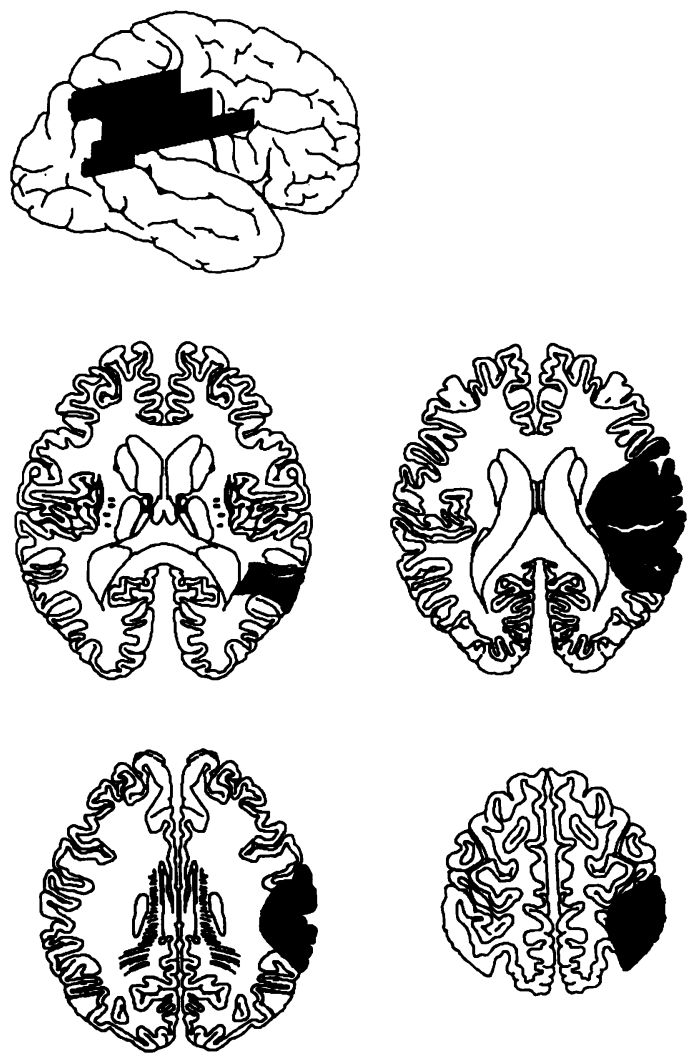

Patient 2

This 72 year old woman was hospitalised because of sudden onset of slurred speech and visual complaints. Examination revealed her to be lucid with normal strength, sensation, and reflexes. Visual fields were intact on confrontation testing with unilateral finger wiggling. The patient showed a clear pattern of neglect on letter cancellation test. CT scan showed a right parietal infarction (figure 1).

Figure 2 Reconstruction of neurological injury of patient 3, showing infarcted area centred in right frontal region.
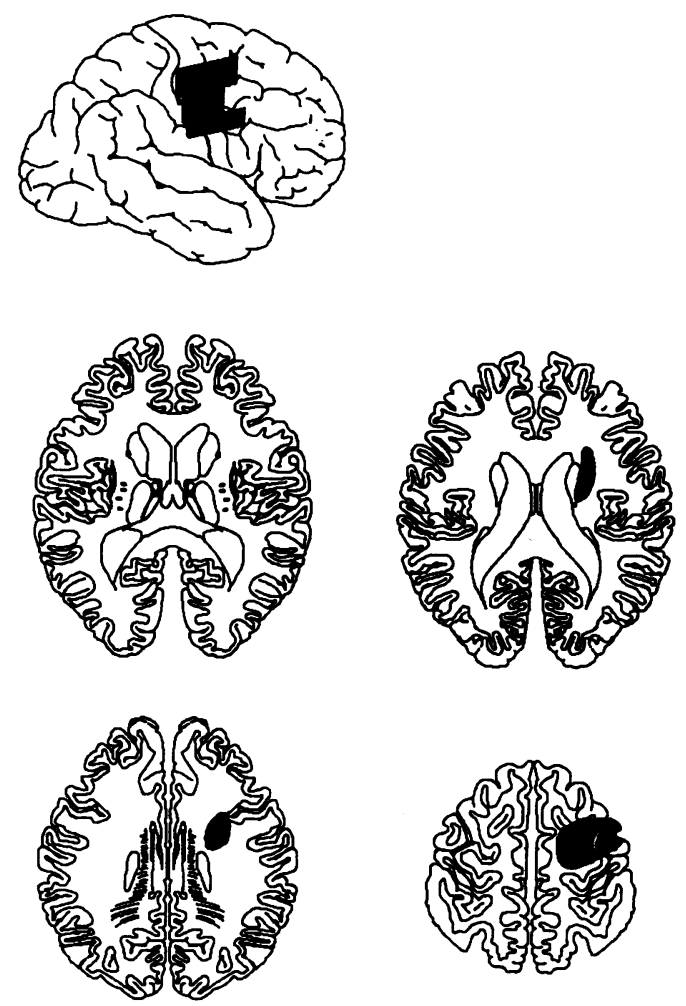

Patient 3

This 41 year old woman was hospitalised because of abrupt onset of left hemiplegia, which developed within an hour after using crack cocaine. Initially the hemiplegia was dense with left sided neglect, a left homonymous hemianopia, absent opticokinetic nystagmus to leftward moving stripes, and left tactile hypesthesia. Three weeks later she was able to wiggle her fingers of her left hand. Touch and pin sensation were intact on the left. Speech was aprosodic, but she was aware of her deficit and had an appropriate appreciation of her circumstances. No hemianopia was present. There was left side extinction to double simultaneous stimulation in both visual and tactile modalities. Line bisection and letter cancellation were normal. Testing for cross modal extinction was conducted and videotaped. CT scan showed an infarct centred in the right frontal region involving the frontal eye fields (figure 2).

Methods of testing for unimodal and cross modal extinction

Patients were tested at the bedside after obtaining informed consent. During the testing for visual extinction the patient was instructed to fixate the nose of the examiner, who performed a conventional "finger wiggle". At a viewing distance of about $120 \mathrm{~cm}$ the finger wiggle appeared about 20 degrees of visual angle in to the right and left visual fields. Unilateral detection in each visual field was tested; extinction was tested with bilateral simultaneous finger wiggle. During the testing for tactile extinction, a light touch was applied to the dorsum of the hands with the patient's eyes closed. Unilateral testing was done for each hand as well as testing for extinction with bilateral simultaneous stimulation. To test for crossmodal extinction the patient was instructed to fixate the nose of the examining neurologist who, on half of the trials, used his left hand to orient visual attention to the right visual hemifield and used his right hand to apply the concurrent tactile stimulus to the patient's right or left hand. On the remaining trials, the left hand was used to orient visual attention to the left visual field and the right hand was used to apply a concurrent tactile stimulus to the patient's left or right hand. The examining neurologist's hand, which applied the tactile stimulus was not visible to the patient on crossmodal stimulation trials. Two types of visual tasks were used when ipsilateral visual stimulus applications were paired with contralateral tactile stimulation. One task required the detection of the examiner's ipsilateral finger wiggle; the second required the counting of the examiner's fingers. On finger counting trials, the examiner raised one to five fingers and the patient was required to report the number of visible fingers. The number of visible fingers varied randomly within the block of trials. Experimental conditions were tested in separate blocks of trials with 15 trials each. All patients were tested on at least one block of 15 trials. Patients 1 and 2 were also tested on a 
Table Results of intra- and cross-modal extinction tests for contralaterally applied stimulus when attention was directed to concurrently applied ipsilateral stimulus. Cell entries show percentage of trials in which extinction occurred; values in parenthesis indicate number of trials per block.

\begin{tabular}{|c|c|c|c|c|c|c|}
\hline & \multicolumn{6}{|c|}{ Contralateral stimulus } \\
\hline & \multicolumn{3}{|c|}{ Visual finger wiggle } & \multicolumn{3}{|c|}{ Dorsal hand touch } \\
\hline & Patient 1 & Patient 2 & Patient 3 & Patient 1 & Patient 2 & Patient 3 \\
\hline \multicolumn{7}{|l|}{$\begin{array}{l}\text { Ipsilateral stimulus } \\
\text { Visual finger wiggle }\end{array}$} \\
\hline $\begin{array}{l}\text { Block 1 } \\
\text { Block } 2\end{array}$ & $\begin{array}{r}100(15 \\
33(15)\end{array}$ & $\begin{array}{l}100(15) \\
100(15)\end{array}$ & $100(15)$ & $0(15)$ & $0(15)$ & $0(15)$ \\
\hline $\begin{array}{l}\text { Visual finger counting } \\
\text { Dorsal hand touch }\end{array}$ & & & & $0(15)$ & $0(15)$ & $0(15)$ \\
\hline $\begin{array}{l}\text { Block } 1 \\
\text { Block } 2\end{array}$ & $0(15)$ & $0(15)$ & $0(15)$ & $\begin{array}{l}100(15) \\
100(15)\end{array}$ & $\begin{array}{l}100(15) \\
100(15)\end{array}$ & $100(15)$ \\
\hline
\end{tabular}

second block of 15 trials with intra-modal extinction trials.

\section{Results}

During the experimental testing none of the patients ever failed to report a unilateral visual or tactile stimulus on either side. As can be seen in the table, all three patients also showed consistent extinction within each modality with simultaneous ipsi- and contralateral stimulus applications. The table also shows the complete lack of cross-modal extinction across all three patients. All patients were aware of tactile sensory input in the contralateral side of their body when a simultaneously presented finger wiggle was applied to (and noticed in) the ipsilateral visual field. All patients also detected contralaterally applied tactile stimulation when the difficulty of the visual task was increased by asking the subject to count the number of fingers presented in the ipsilateral visual field. This lack of cross modal extinction was symmetrical in that orienting attention to the ipsilateral right hand via touch did not prevent the detection of visual stimulus (finger wiggle) in the contralateral left visual field in all three patients.

All three patients also participated in two additional blocks of trials, not shown in the table. On these blocks, the activity of two examiners was coordinated; one examiner applied a short-duration double stimulus tactile stimulation while the secnd examiner simultaneously revealed a specific number of fingers in the patient's contralateral visual field (no ipsilateral visual stimulus was applied). This test was applied to determine whether the shifting of attention to the patient's contralateral visual hemispace would prevent contralateral extinction to bilateral touch stimulation. The results showed that orienting visuospatial attention to contralesional hemispace did not affect the patient's extinction in the tactile modality: all patients reported all ipsilateral touch stimulation but no contralateral touch stimulation (Patient 1 received 20 trials each for ipsi- and contralateral touch stimulation; patients 2 and 3 received 15 trials each in the two conditions.) All patients were also able to report the number of fingers shown in the contralateral visual field.

\section{Discussion}

The three patients reported here were selected to enable a valid and critical test for cross modal extinction between visual and tactile modalities. All patients had reliable extinction in each modality but no primary sensory deficit or mental impairment. To our knowledge there has been no previous report of a formal test for cross modal visual-tactile extinction in patients meeting these criteria. The results clearly showed no evidence of cross-modal extinction either to left visual field stimulation (while simultaneously administering tactile stimuli to the right hand) or to left hand tactile stimulation (while simultaneously reporting signals in the right visual field). Moreover, when testing with double simultaneous tactile stimulation, extinction in the left hand persisted even when the lesioned right hemisphere should have been alerted to the left hemispace by summoning visual attention to the left visual field. Our central findings - that is, lack of cross-modal extinction together with reliable and persistent intra-modal extinction-indicate that functionally autonomous (modality specific) types of spatial attention control the processing of sensory input from tactile and visual modalities, even though both types of modality specific attention are supported by structures in the fronto-parietal lobe system. As such, these results suggest that fronto-parietal structures may not be implicated in the formation of a unified (supra-modal) spatial representation.

We tested whether a stimulus sufficient to cause extinction in one modality was also sufficient to produce cross modal extinction, the critical test for a supra-modal attention mechanism. Possibly, as with the cases of Crichley ${ }^{5}$ and Bender, ${ }^{6}$ an intense stimulus in one modality could cause the failure to report a stimulus in another modality as patients with unilateral fronto-parietal damage and neurologically intact subjects might not notice a doorbell ring if they were to hit a thumb with a hammer simultaneously. The critical test is not whether stimulation in one sensory modality prejudices the ability to attend to another but whether a signal ipsilateral to the lesion, which would be sufficient to cause extinction in the same modality, causes a selective inability to detect a signal in the contralesional field which is in a different modality. 
Two correlational studies examining visual and auditory extinction in parietal patients are consistent with our main conclusions in that they report modality-specific effects of attention. Specifically, DeRenzi et $a l^{7}$ as well as Sieroff and $\mathrm{Michel}^{8}$ found that the presence of visual extinction was not related to auditory extinction. At least one study examining auditory and visual modalities, however, showed cross modal effects of auditory and visual stimulation in patients with parietal lesion. With an attention precuing reaction time paradigm, Farah et $a l^{9}$ replicated the results of Posner et al ${ }^{23}$ in showing that a visual signal in the ipsilesional field causes an "extinction-like reaction time pattern" in which patients with parietal injury were slow to disengage attention to detect visual targets in the contralesional field. In addition, a similar extinction-like reaction time pattern, was obtained when non-predictive auditory cues preceded visual targets, suggesting the involvement of a supra-modal attention system. Consistent with a hierarchical organisation of spatial attention, ${ }^{3}$ Farah et al concluded that a superordinate (unified) control system is used to coordinate shifts of spatial attention across modalities. ${ }^{9}$

In view of their results, it seems possible that clinical examination, as used in the present study, may not be sensitive enough to detect relatively subtle effects of cross-modal extinction. Our complete failure to observe even a single case of cross-modal tactile-visual extinction, however, suggests another possibility: attentional processes may be coordinated across some modalities but not across others.
Specifically, a superordinate unified attentional system may control access to visual and auditory sensory input, given that both types of sensory input generally provide extra-personal information (that is, attention is oriented away from the body). A functionally autonomous type of attention may be used to control the monitoring of tactile sensory input, given that this modality provides peri-personal information (that is-attention is oriented towards the body). This possibility suggests a heterarchial, rather than a hierarchial, structuring of spatial attention systems.

This research was supported by Public Service Grant MH41544 to Robert Rafal and by NICHD grant HD24796 and NSF grant BNS9017880 to Albrecht Inhoff.

1 Posner MI, Cohen Y, Rafal R. Neural systems control of spatial orienting. Philosophical Trans $R$ Soc Lon spatial orienting.

2 Posner MI, Walker JA, Friedrich FJ, Rafal RD. Effects of parietal lobe injury on covert orienting of visual attention Neurosci 1984;4:1863-74.

3 Posner MI, Inhoff AW, Friedrich FJ, Cohen A. Isolating attentional systems: a cognitive-anatomical analysis. Psychobiol 1987;15:107-21.

4 Posner MI, Walker JA, Friedrich FA, Rafal RD. How do the parietal lobes direct covert attention? Neuropsychologia 1987;25:135-46.

5 Critchley M. The parietal lobes. New York: Hafner Press, 1953:146.

6 Bender MB. Disorders of visual perception. In: Halpren L, ed. Problems of dynamic neurology. New York: Grune and Stratton, 1963:344.

7 DeRenzi E, Massimo G, Pattachini F. Auditory extinction following hemisphere damage. Neuropsychologia 1984, 22:733-44.

8 Sieroff E, Michel F. Verbal visual extinction in right/left hemisphere lesion patients and the problem of lexical access. Neuropsychologia 1987;25:907-18.

9 Farah MJ, Wong AB, Monheit MA, Morrow LA. Parietal lobe mechanisms of spatial attention: modality-specific or supramodal? Neuropsychologia 1989;27:461-70. 